\title{
\begin{tabular}{l|l} 
POLITIQUES \& & Politiques et management public
\end{tabular}

\section{Les Pôles de Compétitivité, un nouveau pilotage de la politique industrielle? Bilan en Ile-de-France}

Competitive clusters, a new governance of industrial policy?

Sabine Menu

\section{OpenEdition}

Édition électronique

URL : http://journals.openedition.org/pmp/3325

ISSN : 2119-4831

\section{Éditeur}

Institut de Management Public (IDPM)

Édition imprimée

Date de publication : 15 mars 2011

ISSN : 0758-1726

\section{Référence électronique}

Sabine Menu, «Les Pôles de Compétitivité, un nouveau pilotage de la politique industrielle ? Bilan en |le-de-France », Politiques et management public [En ligne], Vol 28/1 | 2011, mis en ligne le 07 juin 2012, consulté le 01 mai 2019. URL : http://journals.openedition.org/pmp/3325 


\title{
Les Pôles de Compétitivité, un nouveau pilotage de la politique industrielle? Bilan en lle-de-France
}

\author{
Sabine Menu \\ Institut pour l'Entrepreneuriat (GREMA)/Audencia Ecole de Management \\ 8 , route de la Jonelière, 44312 Nantes
}

\section{Résumé}

En 2005, le Gouvernement français lance (selon ses termes) une «nouvelle politique industrielle » portant sur la création de Pôles de Compétitivité répartis sur l'ensemble du territoire national. Réalisant un effort financier de trois milliards d'euros sans commune mesure en Europe, il décide de financer par ce biais des projets de recherche $\&$ développement venant des acteurs économiques privés et sélectionnés par les membres des gouvernances des Pôles. L'analyse de la définition de la politique au niveau central et de sa mise en œuvre en lle-de-France montre cependant les limites de la rupture voulue par cette politique en termes de nouveaux modes de gouvernance. Par la comparaison européenne, en particulier avec le cas bavarois, l'article illustre que la politique des Pôles apparaît en premier lieu comme un instrument d'action publique de l'Etat central pour se mettre en scène une nouvelle fois comme un acteur clé du champ industriel.

(c) 2011 IDMP/Lavoisier SAS. Tous droits réservés

Mots clés : pôles de compétitivité, politique industrielle, Ile-de-France, Bavière, modes de gouvernance.

\section{Abstract}

Competitive clusters, a new governance of industrial policy? Results in lle-de-France. In 2005, the French Government launched what was called a « new industrial policy » aiming at creating Competitive clusters (Pôles de Compétitivité) which were distributed in all the regions. Investing up to three billion Euros, which has been a considerable amount of money if compared to other European countries, the goal has been to support research and development collaborative projects coming from, and selected by the private sector in the Pôles' governance. The article presents the policy definition process at the central level, and the implementation process in the region Ile-de-France (Paris region). It shows

\footnotetext{
*Auteur correspondant : smenu@audencia.com doi:10.3166/pmp.28.105-121 @ 2011 IDMP/Lavoisier SAS. Tous droits réservés
} 
the limited effects of the introduction of the new modes of governance. Thanks to the European comparison, in particular with Bavarian cluster policy, it outlines that the Pôles have been first of all a tool for the French Government for a new "mise en scène" of its role as a key actor in the industry.

(c) 2011 IDMP/Lavoisier SAS. Tous droits réservés

Keywords : competitive clusters, industrial policy, lle-de-France, Bavaria, modes of governance

En juillet 2005, le Gouvernement de Villepin lance la politique des Pôles de Compétitivité qui s'est traduite depuis par la création d'environ 70 Pôles et par un engagement public totalisant trois milliards d'euros. Dans les rapports au Gouvernement consacrés à l'industrie (Blanc, 2004 ; Datar, 2004 ; Beffa, 2005), l'action de l'Etat est justifiée pour, d'une part, répondre aux risques de «désindustrialisation » dans un contexte de mondialisation accélérée ; d'autre part, mettre en œuvre une politique qui réponde à l'engagement pris par la France avec ses homologues européens en 2000 de réaliser des efforts en vue de soutenir la compétitivité et l'innovation de l'économie européenne dans le cadre de la stratégie de Lisbonne. Ces rapports concluent ensuite à des moyens d'action différents, l'un préconisant une politique industrielle par « grands projets technologiques » (Beffa, 2005) alors que d'autres soutiennent une approche par clusters (Blanc, 2004) et par les territoires (Datar, 2004). Au final, le Gouvernement choisit de favoriser par son action le développement de clusters, c'est-à-dire la concentration d'entreprises qui nouent des relations de collaboration entre elles et avec d'autres acteurs privés (centres de recherche...) et publics pour développer leurs activités d'innovation dans un domaine et sur un territoire donnés (Porter, 1998).

L'évaluation scientifique de la politique des Pôles a jusqu'à présent été réalisée en suivant une approche fonctionnaliste, c'est-à-dire qu'elle a porté sur l'analyse de ce que l'on peut attendre d'une intervention publique dans le développement d'un cluster ou sur l'observation de ses résultats socio-économiques ou organisationnels. Elle a cherché aussi à tempérer l'enthousiasme exprimé dans les rapports publics. Ainsi, Duranton et al. (2008) estiment à partir de l'étude d'une politique précédente, les Systèmes Productifs Locaux (SPL), que l'on peut s'attendre à un effet économique limité des Pôles. En sociologie, à partir de l'analyse détaillée du Pôle aéronautique toulousain, Grossetti et Zuliani (2009) estiment que c'est surtout la succession de politiques publiques dans le temps long qui produit des effets sur les collaborations au sein des clusters. En gestion, les origines et les premières expériences des Pôles ont été décrites (Weil et Fen Chong, 2008) ainsi que la portée des gouvernances des Pôles pour développer des clusters (Gomez, 2009).

Dans cet article, ce sont les logiques de l'action publique qui sont analysées pour montrer en quoi la politique des Pôles est «nouvelle » et comprendre ce qu'elle produit. En particulier, il pose la question de ce que produisent les gouvernances des Pôles qui sont placées au cœur du nouveau dispositif d'action publique. Quelles formes prennent ces collaborations, c'est-à-dire quels en sont les acteurs, les niveaux d'action et les résultats en termes de pilotage des Pôles? En s'appuyant sur l'approche par les « instruments d'action publique » (Lascoumes et Le Galès, 2006), l'objectif de cet article est de montrer que les Pôles ne sont pas des dispositifs techniques neutres, mais des outils qui ont été retenus, par rapport à d'autres, pour produire des changements organisationnels et normatifs dans la 
structuration du champ industriel. En particulier, les Pôles ont été décidés et mis en œuvre selon des techniques issues du management privé et introduites dans l'appareil d'Etat pour renforcer l'action centrale. Il apparaît alors, aux vues aussi de la comparaison avec les politiques de cluster menées en Europe et en particulier avec le cas bavarois, que les Pôles sont en premier lieu un instrument de l'Etat centralisé pour se repositionner comme un acteur clé du champ industriel.

Les logiques et les effets de la mise en œuvre de la politique des Pôles sont illustrés à partir des résultats d'une enquête empirique réalisée en Ile-de-France en 2009. Parce qu'ils visent à soutenir des collaborations dans un domaine et un territoire donnés, les Pôles s'analysent au mieux à l'échelle d'une région et non de la France. Il existe ensuite plusieurs intérêts à étudier la première région économique en Europe et la « région-capitale ». L'Ilede-France concentre en effet non seulement les pouvoirs politiques et administratifs français, mais aussi en termes économiques (28\% PIB national) - notamment industriel (Berthet et Ternisien, 2004) - et de recherche (première région européenne avec 3\% du PIB consacré à la recherche \& développement - R\&D - dans les années 2000). Ceci la place d'emblée dans une perspective de compétition internationale (Crespy, 2007) et fait de l'innovation en Ile-de-France une question traitée par les politiques nationales (Gilli et Offner, 2009). De plus, c'est une région qui est restée longtemps éloignée des transformations produites par la décentralisation, dont témoigne le contrôle de l'aménagement du territoire par l'Etat central ou encore une alternance politique régionale tardive (1998). Du fait de ces enjeux industriels ou de recherche et de la récente émergence d'une action politique régionale, l'Ile-de-France constitue un terrain pertinent sur les modes de gouvernance des Pôles. L'enquête a été menée à partir d'une revue de la littérature sur les collaborations d'acteurs publics et privés dans l'industrie francilienne, l'étude de documents des organisations publiques et privées au niveau central, régional et local impliquées dans les Pôles, et la conduite d'entretiens semi-directifs auprès d'acteurs et de responsables publics et privés impliqués dans la politique des Pôles ${ }^{1}$.

\section{Les Pôles ou la recomposition de l'action étatique centralisée dans l'industrie}

\subsection{La politique des Pôles, une action publique décidée et organisée au niveau central}

La politique industrielle française est traditionnellement considérée comme atypique en Europe. Ce n'est pas tant le niveau élevé des dépenses publiques (tous les Etats européens engagent des ressources importantes pour venir en aide à l'industrie), mais l'intervention directe de l'Etat dans l'économie qui a retenu l'attention. En France, cette intervention est en outre rendue bien visible car pilotée (et publicisée autour de la « rhétorique industrialiste » pour affirmer la souveraineté de l'Etat, Cohen, 1992a) par un nombre restreint d'acteurs. La politique industrielle de l'Etat a ainsi été longtemps qualifiée de « dirigiste » du fait notamment des relations privilégiées nouées au niveau central et de manière directive entre hauts-fonctionnaires, dirigeants indus-

\footnotetext{
${ }^{1}$ Entretiens auprès de responsables de la DGCIS, OSEO/Ile-de-France, Direction Régionale de I'Industrie, la Recherche et les Entreprises (DRIRE), Conseil Régional de I'lle-de-France, Agence Régionale de Développement, une conseillère régionale de la majorité, un chargé de mission du Conseil Général des Yvelines, deux chargés de mission de la Mairie de Paris, un responsable de Cap Digital.
} 
triels et élus politiques (Schmidt, 1996). Conserve-t-elle ce caractère atypique avec les Pôles ? Schmidt (1997) conclut au déclin du dirigisme industriel français, mais les nombreux rapports adressés au Gouvernement dans la phase de définition de la politique des Pôles et invoquant le retour du volontarisme politique de l'Etat (la Datar parle en 2004 d'une «nouvelle politique industrielle »), de même que l'idée véhiculée à l'étranger des Pôles comme n'étant qu'une nouvelle façon pour l'Etat central d'intervenir directement dans l'économie et de contourner la réglementation européenne (Duranton et al., 2009), amène à s'interroger sur sa réémergence.

Par contraste avec la période des nationalisations et des grands projets issus de la planification, l'action publique dans le champ industriel au cours des années 80 et 90 s'oriente vers un soutien à la compétitivité des entreprises, et ce, pour les Gouvernements successifs, au nom de la « contrainte européenne » du Marché Unique (Cohen, 1992a). Au sein de l'appareil d'Etat, le Ministère de l'Industrie est supprimé en 1997 et est remplacé par un Secrétariat. Au début des années 2000, le Ministre délégué à l'Industrie est uniquement en charge des secteurs de l'énergie, des matières premières et de l'économie numérique (télécoms). Il est rattaché au Ministère de l'Economie et des Finances. Les autres secteurs industriels sont suivis par des ministères techniques (Défense, Agriculture, Equipement, Santé...).

Lancée en 2005, la politique des Pôles devient un instrument de recomposition et de renforcement de l'action de l'Etat dans le champ industriel. Au début des années 2000, outre les rapports déjà cités qui confrontent différentes approches du soutien industriel, la notion de «politique industrielle » est de plus en plus utilisée au plus haut sommet de l'Etat. Ainsi, d'après une étude de la presse quotidienne nationale entre 2003 et 2005, on constate que dans plus de la moitié des articles (54\%) l'expression est utilisée par le Chef de l'Etat, dans près de 40\% par le Premier Ministre et dans 6\% des cas seulement par le Ministre de l'Industrie ${ }^{2}$. Différentes agences sont créées au niveau central, qui traduisent cet engouement pour le soutien à l'industrie et à l'innovation : l'Agence de l'innovation industrielle (AII), l'Agence Nationale de la Recherche (ANR), ou encore OSEO (structure née du rapprochement entre l'Agence Nationale pour la Valorisation de la rechercheANVAR - et de la Banque pour le Développement des Petites et Moyennes Entreprises $(P M E)$ - BDPME, à quoi s'ajoute une fusion avec l'AII en 2007).

De plus, c'est bien au niveau de l'Etat central qu'est décidée la politique des Pôles. Après des négociations longues liées, d'une part, à des représentations opposées sur le type et le niveau d'action à activer (illustrées par les rapports de Blanc, Beffa et de la DATAR) et, d'autre part, aux tensions internes au Parti de droite UMP au pouvoir, en particulier entre régionalistes (le Premier Ministre J-P. Raffarin) et centralistes (le Ministre de l'économie, puis de l'Intérieur N. Sarkosy), le Comité Interministériel d'Aménagement du Territoire de juillet 2005 décide de financer des Pôles de compétitivité à hauteur d'1,5 milliards d'euros pour trois ans (2005-2008) $)^{3}$. Il s'agit donc d'une politique industrielle à visée

\footnotetext{
${ }^{2}$ Annexe du rapport d'information parlementaire sur Les outils de la politique industrielle, étude conduite par la Compagnie européenne d'intelligence stratégique à partir d'une analyse quantitative de 842 articles contenant l'expression « politique industrielle » parus dans la presse quotidienne nationale entre janvier 2003 et mai 2005 (rapport présenté par M. Carayon le 10 mai 2005 à l'Assemblée Nationale).

${ }^{3}$ Cette somme correspond à l'ensemble des aides aux Pôles, y compris les subventions de l'Agence nationale de la recherche, du Fonds de compétitivité des entreprises, de la Caisse des Dépôts et Consignations et d'Oseo (ainsi que de l'Agence de l'innovation industrielle avant sa fusion avec Oseo en 2007).
} 
microéconomique pour soutenir les projets de $R \& D$ des acteurs économiques privés situés sur le territoire français. 71 Pôles en résultent jusqu'en 2009, partagés en trois catégories : une dizaine de «Pôles mondiaux », une quinzaine de «Pôles à vocation mondiale » et le reste correspondant aux «Pôles nationaux » (Weil et Fen Chong, 2008). Comme l'indique la carte ci-dessous, les Pôles se répartissent sur l'ensemble du territoire national, soit un Pôle au moins par région ; mais en termes financiers, plus de la moitié des fonds (55\%) est consacrée aux 11 Pôles mondiaux (Boston Consulting, 2008). Il s'agit donc, avec la création des Pôles, non pas d'une nouvelle mesure d'aménagement du territoire du Gouvernement français mais bien d'un soutien à des projets de R\&D.

\section{Carte - La répartition des Pôles de Compétitivité en 2009}

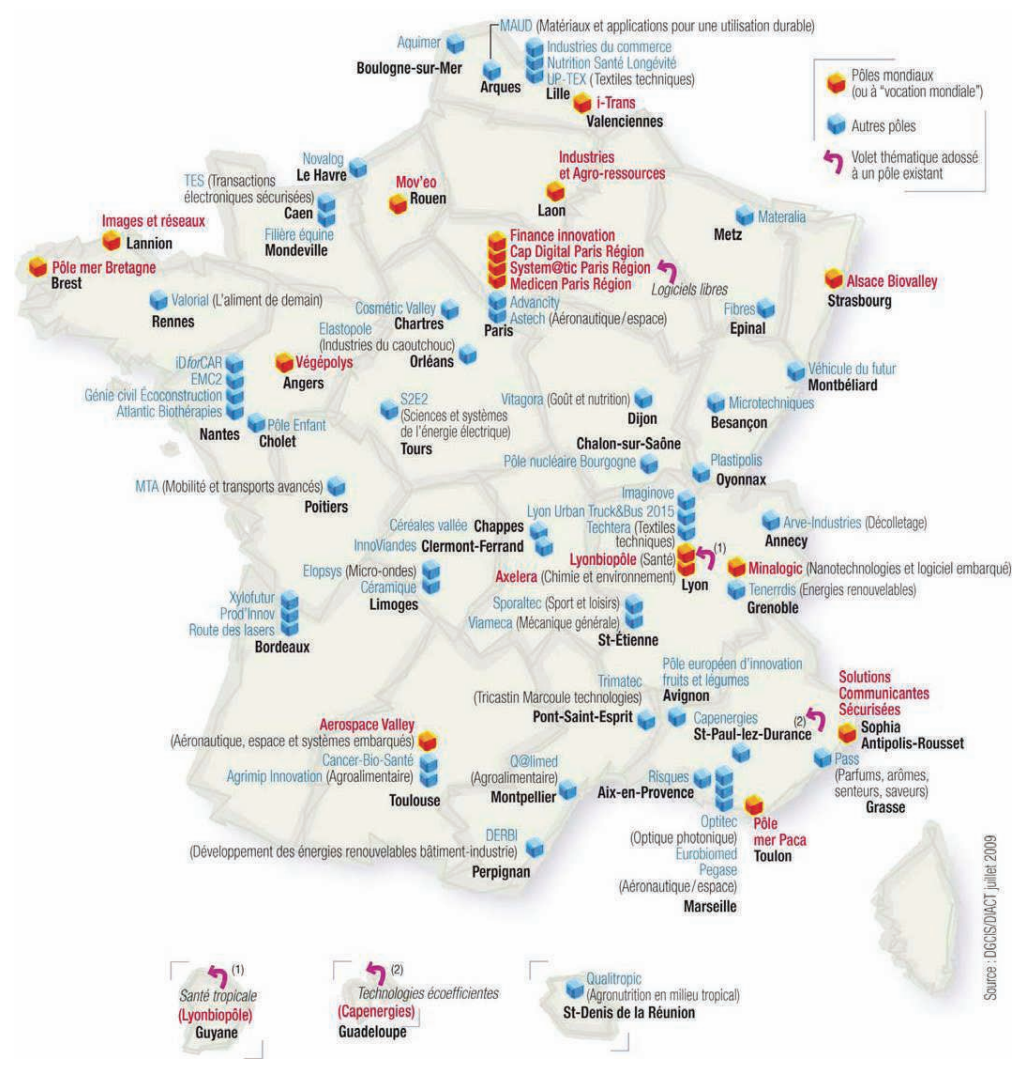

Source - DGCIS/DIACT (juillet 2009)

La mise en œuvre de la politique des Pôles de Compétitivité se fait également de manière centralisée. D'une part, elle est sous la conduite de la Délégation à l'Aménagement du Territoire et à l'Action Régionale (DATAR), rebaptisée pour l'occasion Délégation Interministérielle à l'Aménagement et à la Compétitivité des Territoires (DIACT), et qui est rattachée directement au Cabinet du Premier Ministre ; et de la Direction Générale à la Compétitivité, à l'Innovation et aux Services (DGCIS) créée en janvier 2009 et qui remplace la Direction Générale des Entreprises (DGE). Des agents de contact sont créés 
pour chaque Pôle au sein de la DGCIS. D'autre part, l'engagement financier est organisé au sein d'un Fonds interministériel de Compétitivité des Entreprises (FCE), et complété par les ressources de l'ANR, OSEO, ainsi que la Caisse des Dépôts et Consignations.

En outre, les Pôles sont sélectionnés selon une procédure unique de candidature pilotée et financée par les Administrations centrales. Pour obtenir le label de «Pôle », il ne faut pas prouver l'existence d'un cluster mais proposer sa candidature autour d'un projet stratégique lors des appels lancés par le Gouvernement. La procédure d'appels à candidature est fondée sur différents critères définis par l'Etat central (projets innovants collaboratifs, focalisation thématique, acteurs intervenant à différents niveaux de création de valeur...), et elle est encadrée par la sélection organisée au niveau étatique, dans un premier temps par les Préfectures de Région puis, au niveau central, par un groupe d'experts auprès des Ministères et un groupe de personnalités qualifiées indépendantes. Dans la seconde phase de la politique des Pôles engagée depuis 2008, l'Etat demande aux Pôles de rédiger une «feuille de route stratégique » suivant des critères fixés par lui. Leur élaboration est marquée par de nombreux allers-retours entre le Groupe de Travail Interministériel (GTI) et les Pôles.

\subsection{Les Pôles, la décentralisation et les techniques managériales de l'Etat : le renforcement de l'action centrale}

La politique des Pôles s'inscrit et appuie deux autres chantiers politiques et institutionnels qui viennent renforcer l'action centrale de l'Etat. D'un côté, elle entre dans le « jeu de compromis » (Le Lidec, 2007) qui existe entre le pouvoir central et les collectivités territoriales dans les négociations sur les nouvelles étapes de la décentralisation. Comme souligné précédemment, il existe un conflit entre centralistes et régionalistes au sein du Parti en place à la tête du pouvoir. La Réforme Constitutionnelle de 2003 fait des Régions des collectivités territoriales à part entière ${ }^{4}$ et la loi ordinaire l'accompagnant en 2004 définit les Régions comme les «chefs de file du développement économique ». Or, la décision est prise de ne pas faire participer officiellement les Régions aux Pôles (comme le notent Weil et Fen Chong en 2008, le résultat des élections de mars 2004 n’y est pas étranger avec 20 Régions sur 22 qui sont désormais gouvernées par une majorité de gauche). L'Etat laisse les Régions libres de s'y engager - ce qu'elles font activement, comme cela sera montré dans le cas francilien. En retour, les Régions vont exercer leur lobbying pour avoir une répartition des Pôles sur l'ensemble de la France. Aucun Président de Région, quelle que soit sa couleur politique, ne s'oppose aux Pôles ; en d'autres termes, il n'y a pas de discussion sur le choix de l'instrument d'action publique des Pôles, mais plutôt sur la répartition de ses gains potentiels.

De l'autre, l'engagement en faveur des Pôles de compétitivité devient un moyen pour l'Etat de se «moderniser » par le biais de la réforme de l'administration publique et de l'introduction de techniques de management (Albertini, 2007 ; Bezes, 2009). Dans la lignée de la réforme engagée par la LOLF (Loi organique relative aux lois de finances) visant à appliquer à l'action de l'Etat les principes du New Public Management (Bezes, 2009), la DGCIS voit avec la politique des Pôles le renforcement du pouvoir de son directeur, Luc Rousseau, qui est l'ex-Directeur Général des Entreprises et qui a déjà été fortement impliqué

\footnotetext{
${ }^{4}$ Loi constitutionnelle du 28 mars 2003 sur l'Organisation décentralisée de la République Française.
} 
dans les préparatifs de cette politique en tant que conseiller du Premier Ministre Raffarin entre 2003 et 2005. Par son expertise, Rousseau joue un rôle de premier ordre au sein du GTI qui est en charge de la politique des Pôles. Plus généralement, ce groupe « devient le lieu de régulation technique mais aussi d'élaboration d'une doctrine partagée par les différents ministères et soumise à l'approbation du pouvoir politique » (Albertini, 2007).

De plus, la définition, la mise en œuvre et l'évaluation de la politique des Pôles suivent les principes et les techniques du management. Ceci n'est pas nouveau dans l'action de l'Etat, mais on observe sa systématisation dans le cadre de la LOLF en général (Bezes, 2009) et de la politique des Pôles en particulier. Ainsi, des appels à candidature sont organisés régulièrement pour sélectionner des projets posés en concurrence. Les Pôles obtiennent un label et sont évalués en fonction d'un benchmarking (ou étalonnage) international. Les critères d'évaluation sont régulièrement reformulés pour améliorer la performance de la politique en termes de nombre de projets collaboratifs, de brevets déposés... et à défaut de l'évaluer selon les résultats économiques qui sont encore difficiles à obtenir, ou de la confronter à des objectifs de développement économique à plus long terme. Le recours à l'expertise externe est aussi une technique empruntée au management pour légitimer l'évaluation en termes de «performance » de l'action menée. Officiellement, ce n'est d'ailleurs qu'aux vues des conclusions du rapport commandé par le Gouvernement Fillon en 2008 aux consultants Boston Consulting Group et CM International que les financements de la politique des Pôles sont renouvelés pour 2009-2011.

Or, ces techniques du management ne sont pas de simples outils d'évaluation, mais elles sont porteuses des valeurs du privé (gestion par objectifs, concurrence, culture de la performance...), de même qu'elles constituent un moyen pour l'Etat central de contrôler le processus de mise en œuvre de la politique, de lui fixer des étapes, de discipliner les acteurs (Bruno, 2008) - au dépend y compris des représentants de l'Etat en Région ${ }^{5}$ qui se voient en outre imposés une refonte de leur service dans le cadre de la $\mathrm{LOLF}^{6}$. Mis ensemble, ces trois processus liés à l'introduction des Pôles (action centralisée, négociations avec les collectivités territoriales, réformes managériales) vont dans le sens d'une recomposition et d'un renforcement de l'action étatique centrale dans le champ industriel, tant du point de vue organisationnel, financier que normatif.

\section{La mise en cuvre des Pôles en lle-de-France : portée et limites des modes de gouvernance}

L'Ile-de-France est l'une des 22 régions françaises mais d'un point de vue économique et politique, elle n'est pas une région comme les autres. Toutefois, ses particularités en font un terrain d'étude pertinent au moment où l'Etat décide de financer les Pôles selon un critère, non pas d'aménagement du territoire, mais de compétitivité et d'attractivité économique. Pour le résumer en deux données, ce qui pose " problème » aux responsables politiques dans cette région, c'est qu'elle rassemble un potentiel de recherche inégalé en Europe mais se situe seulement à la neuvième place des régions européennes en termes d'innovation. La politique des Pôles ouvre de nouvelles pers-

\footnotetext{
${ }^{5}$ Entretien personnel avec un responsable de la DRIRE de I'lle-de-France (2009).

${ }^{6}$ La réforme de I'Etat fait fusionner les DRIRE avec d'autres Directions régionales (DREAL, DIRECCTE, DRCE, DRCCF...).
} 
pectives financières pour cette région. En effet, l'Ile-de-France a été longtemps tenue à l'écart des aides de la politique industrielle liées à l'aménagement du territoire, tant au niveau de l'Etat français que de celui européen, et en dépit de ses inégalités socioéconomiques internes ${ }^{7}$. Elle est la région française la moins bien traitée dans l'élaboration des Contrats de Plan Etat-Région de 2000-2006 ${ }^{8}$, et le financement prévu pour 2007-2013 reste encore dissymétrique ${ }^{9}$.

\subsection{L'ouverture du jeu des acteurs du champ industriel}

L'Etat central est légitimé pour intervenir sur les questions de compétitivité et d'innovation du fait du caractère d'exception du territoire francilien. Il intervient traditionnellement dans les décisions d'aménagement et de développement économique : en témoigne son droit de veto dans les plans d'aménagement franciliens ou ses Opérations d'Intérêt National - comme par exemple sur le Plateau de Saclay (Brédif, 2009). Mais depuis la décentralisation, les collectivités territoriales le sont aussi. Certains ont affirmé au moment du lancement des Pôles que ces derniers étaient un «électrochoc pour les régions » et permettaient une "prise de conscience, par tous les acteurs, de la réalité économique de leur territoire et des besoins des entreprises » (Pascallon et Hortefeux, 2008).

Or, les régions ont constitué depuis les années 80 à travers l'Europe des espaces d'action où le jeu des acteurs s'est ouvert. Sous l'effet des transformations du système économique capitaliste, de l'accélération de l'intégration européenne, le renforcement de la politique régionale au niveau communautaire et des politiques de régionalisation ou de décentralisation au sein des Etats-membres, des coalitions d'acteurs variés, issus des secteurs public et privé se sont formées pour définir des solutions territorialisées à ce qui leur est apparu comme des défis industriels ; et, en France, les Régions, en tant qu'institutions, ont participé au développement économique de leur territoire, malgré des ressources et des compétences limitées (Menu, 2009). En 2005, elles considèrent les Pôles comme une fenêtre d'opportunité pour soutenir l'innovation, en lien avec l'Etat, et pour asseoir le rôle qui leur a été donné par la réforme constitutionnelle de 2003. Elles ne sont pas de simples exécutants d'une politique nationale mais y consacrent des moyens financiers (en particulier pour des études) et logistiques importants, et interviennent concernant les choix et les modalités d'intervention dans les projets (Fen Chong, 2006). Au total, ce sont plus de 300 millions d'euros que les collectivités territoriales mettent à la disposition des Pôles, en particulier pour en financer l'animation (Lefebvre, 2009).

Dans les Régions françaises, la cause des Pôles est donc rapidement acquise. Cette cause, bien présente même si elle est plus récente en Ile-de-France, fait en outre l'objet d'une stratégie complémentaire et nouvelle au niveau de l'action publique régionale : d'une part, la Région de l'Ile-de-France propose une politique régionale visant à soutenir la recherche ; d'autre part, elle définit les Pôles comme les « courroies d'entraînement » d'un autre type d'initiative cette fois régionale, celle des filières industrielles.

\footnotetext{
${ }^{7}$ L'Ile-de-France touche pour la première fois en 1998 des aides européennes pour ses territoires urbains en difficulté (Fonds Social Européen).

${ }^{8}$ Elle connaît la dotation par habitant la plus faible et la part de financement régional la plus élevée en France.

${ }^{9}$ Les collectivités franciliennes doivent couvrir 63\% du financement du Contrat de projet 2007-2013.
} 
Ainsi, l'action du Conseil Régional de l'Ile-de-France est récente (sa première « politique de cadrage pour la recherche et l'innovation » date de 2004) mais elle n'en reste pas moins importante. La Région débourse plus de 90 millions d'euros de 2005 à 2009 en budget d'investissement pour la mise en œuvre des Pôles. En outre, alors que le domaine de la recherche n'entre pas dans le champ de ses compétences, elle lance dès 2006 la politique des « Domaines d'Intérêts Majeurs » (DIM). Il s'agit d'un soutien à la recherche, dont celle fondamentale, pour renforcer les coopérations entre les différents pôles de recherche franciliens publics ou semi-publics, en complément des aides aux Pôles. En 2009, la Région consacre ainsi plus de 24 millions d'euros (en investissement) et 17 millions d'euros (en fonctionnement) à cette politique ; soit désormais un montant total annuel supérieur à celui consacré aux Pôles. De plus, en tant que Région gouvernée par une majorité réunissant des partis de gauche et les Verts, elle cherche à se démarquer politiquement en soutenant en particulier les éco-technologies.

Pour soutenir le développement de ce nouvel axe de l'action publique régionale, les services de l'administration régionale qui se consacrent à l'innovation et au développement économique sont réorganisés : d'un côté, la Direction Recherche, Innovation \& Enseignement Supérieur est séparée du Développement Economique \& Emploi et des moyens d'action conséquents en termes financier et du personnel lui sont allouée ; de l'autre, une agence est créée en 2006 pour la promotion économique régionale et fait des Pôles un instrument privilégié de cette promotion. De plus, la Région, avec la DRIRE, développe une stratégie régionale incluant les Pôles dans une réflexion plus large de développement économique : en 2008, les deux organes régionaux élaborent le Plan Filières pour définir une stratégie d'action pour les secteurs industriels prioritaires de la région. Les deux instances y font des Pôles les «courroies d'entraînement » de l'économie francilienne dans son ensemble.

En revanche, dans la phase de sélection des Pôles, la Région de l'Ile-de-France se range derrière deux types d'expertise : celle de l'Etat et son soutien ancien à certains secteurs industriels - défense - ou certaines zones, comme le plateau de Saclay ; et celle d'industriels issus des plus grandes entreprises et centres de recherche franciliens et réunis depuis 2004 au sein du Groupe Olivier (Fen Chong, 2006). Les Chambres de Commerce et d'Industrie et les syndicats, qui constituent des partenaires classiques du développement économique territorial, sont pour leur part tenus à l'écart de la sélection des Pôles.

La mise en œuvre initiale des Pôles en Ile-de-France montre que le choix se porte à la fois sur des secteurs économiques dans lesquels l'Etat est traditionnellement présent - défense, électronique... avec System@tic, l'aéronautique avec AsTech, la santé avec Medicen et l'automobile avec Move'o ; et sur de nouveaux domaines, comme la finance (Finance Innovation), les contenus numériques (Cap Digital) et la mobilité durable (Advancity), qui reçoivent le soutien de la Région de l'Ile-de-France. Trois pôles mondiaux sont définis dans la région (System@tic, Finance Innovation et Medicen) et reçoivent une part importante du FUI. Dans l'ensemble, ce sont près de 120 millions d'euros qui sont accordés aux Pôles franciliens en 2006-2007 ${ }^{10}$. Le tableau 1 présente les Pôles créés en Ile-de-France depuis 2005.

\footnotetext{
${ }^{10}$ Ces chiffres ne tiennent pas compte des Pôles AsTech et Finance Innovation qui sont créés en 2007 : Tableaux de bord des Pôles de compétitivité élaborés par la DGCIS/INSEE/DIACT, édition 2008.
} 
Tableau 1 - Les Pôles franciliens en 2009

\begin{tabular}{|c|c|c|c|c|c|c|c|}
\hline Kon dii plle & $\begin{array}{l}\text { Bate de: } \\
\text { labelisation } \\
\text { [CucI) }\end{array}$ & $\begin{array}{c}\text { Demaine } \\
\text { decomplataces }\end{array}$ & $\begin{array}{l}\text { Nombre } \\
\text { de PME } \\
\text { arfistealtes }\end{array}$ & $\begin{array}{l}\text { Nonbre } \\
\text { degrants } \\
\text { groupes } \\
\text { adhereats }\end{array}$ & $\begin{array}{l}\text { Momble de } \\
\text { buoratuires ou } \\
\text { deteblissements } \\
\text { denseignemein } \\
\text { adherents }\end{array}$ & $\begin{array}{l}\text { Nonibre: } \\
\text { de proists } \\
\text { finances par } \\
\text { des fonis } \\
\text { pithlics }\end{array}$ & 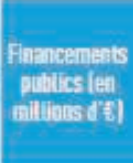 \\
\hline Finance inneratian & $\begin{array}{l}\text { 5juillet } \\
\text { 2007 }\end{array}$ & $\begin{array}{l}\text { Bancues et entreprises } \\
\text { d'investissement, sociétés } \\
\text { d'assurance, secietés de } \\
\text { gestion et capital imes } \\
\text { tissement, sevices aix } \\
\text { entreprises financieres }\end{array}$ & 47 & 105 & 26 & 9 & 7,0 \\
\hline Medicen Paris Ritgioa & $\begin{array}{l}\text { 12juillet } \\
2005 .\end{array}$ & $\begin{array}{l}\text { Hautes technologies } \\
\text { pour la santé }\end{array}$ & 93 & 12 & - & 12 & 30,4 \\
\hline $\begin{array}{l}\text { Pystangitic } \\
\text { Paris rígion }\end{array}$ & $\begin{array}{l}12 \text { juillet: } \\
\text { 20155 }\end{array}$ & $\begin{array}{l}\text { Lagiciels et systemes } \\
\text { complezes pour les } \\
\text { tetecoms, automotile et } \\
\text { transport, sécurité } \\
\text { et defense }\end{array}$ & 183 & 107 & 79 & 117 & 235,4 \\
\hline $\begin{array}{l}\text { Cap nigital } \\
\text { Paris Réggiont: }\end{array}$ & $\begin{array}{c}12 \text { juillet } \\
2005\end{array}$ & $\begin{array}{c}\text { TIC. industries culturelles } \\
\text { numériques. }\end{array}$ & 231 & 20 & 3! & 55 & 25,1 \\
\hline $\operatorname{Mix}^{\prime} \mathrm{E0^{ \circ }}$ & $\begin{array}{l}12 \text { juillet } \\
2005\end{array}$ & $\begin{array}{l}\text { Automobile, transports } \\
\text { collectís,s, sécurite routière } \\
\text { et emvironnement }\end{array}$ & 47 & 51 & 41 & $n$ & 11.9 \\
\hline $\begin{array}{l}\text { Adraicity (a Ville } \\
\text { et mobitite durables) }\end{array}$ & $\begin{array}{l}\text { 12 juillet } \\
2005\end{array}$ & $\begin{array}{l}\text { Déreloppement durable } \\
\text { de lavile, habitat } \\
\text { et construction, } \\
\text { mobilité en milieu urbain, } \\
\text { Eco-technologies }\end{array}$ & 46 & 9 & 19 & 10 & 10,1 \\
\hline ASTechi & $\begin{array}{l}5 \text { juillet } \\
2007\end{array}$ & $\begin{array}{l}\text { Wiation d'affaires, trans- } \\
\text { port spatial, snotorisation } \\
\text { ef equipements }\end{array}$ & 49 & 15 & 23 & 10 & 21,6 \\
\hline Cosmetic velley & $\begin{array}{l}\text { 12juillet } \\
\text { 70015. }\end{array}$ & Parfumefie, cosmétique & 131 & 33 & 17 & 12 & 29,5 \\
\hline Elestopale: & $\begin{array}{l}5 j \text { juilet } \\
2007\end{array}$ & $\begin{array}{l}\text { Industria du caoutchouc et } \\
\text { des polymeres }\end{array}$ & 40 & 10. & 12 & - & - \\
\hline
\end{tabular}

Source - Bilan économique de la Région de l'Ile-de-France $(2009)^{H I}$.

\subsection{La difficile opérationnalisation des gouvernances des Pôles}

Dans ce contexte d'action impliquant les niveaux national et infranational, la politique des Pôles de compétitivité fait de ce qui est appelé la « gouvernance du Pôle » le moteur de la sélection et de la mise en œuvre des projets collaboratifs de R\&D. Les Pôles adoptent une structure associative avec des collèges représentant l'industrie et la recherche. Ils sont régulés par des contrats-cadres qui définissent leurs priorités stratégiques et les implications

\footnotetext{
${ }^{11}$ Les Pôles Cosmetic Valley et Elastopole ne sont pas situés dans la région francilienne.
} 
des différents partenaires. Dès les premières années, elles deviennent opérationnelles au sens où plus de 245 projets sont financés en Ile-de-France (pour un total de 650 en France).

Mais plusieurs éléments empêchent d'observer la stabilisation de modes de gouvernance des Pôles en Ile-de-France, c'est-à-dire « une articulation stabilisée de régulations (dans le mode de coordination des acteurs et/ou des activités, dans l'allocation des ressources et dans la structuration des conflits) »(Le Galès, 2006). Premièrement, la liaison entre la politique nationale des Pôles et l'action économique régionale ne se fait pas sans blocage. Ainsi, d'un côté, dans le cadre du Plan Filières, le Conseil Régional et la DRIRE font des Pôles les courroies d'entraînement de l'économie francilienne dans son ensemble. Mais, de l'autre, quand la Région décide de fixer des priorités politiques dans son soutien aux Pôles (comme les éco-technologies) ${ }^{12}$, elle affronte l'opposition à la fois des représentants de l'Etat en Région et des autres collectivités franciliennes, y compris celles dirigées par une majorité de gauche comme la Ville de Paris, qui lui reprochent de rechercher surtout un «affichage politique $»^{13}$.

Dans une région où les enjeux politiques et économiques sont exacerbés, les conflits de pouvoir s'expriment donc entre des collectivités territoriales puissantes (dont les élus sont aussi souvent des personnalités politiques nationales de premier plan), qu'il s'agisse des villes, départements ou du Conseil Régional. Or, les structures associatives des Pôles, outre le fait qu'elles posent des limites en termes d'autonomie financière vis-à-vis de l'Etat et des grands groupes, sont trop faibles d'un point de vue organisationnel pour pouvoir réguler ces conflits. Il en résulte que les Pôles sont dépendants de leurs acteurs les plus puissants (les grands groupes) et de leurs financeurs, l'Etat et les collectivités territoriales, ces dernières étant soucieuses avant tout du développement économique local dont elles ont la charge.

Deuxièmement, le bilan de la participation des acteurs économiques privés à la gouvernance des Pôles franciliens est mitigé. Les Pôles sont dominés par les grandes entreprises et centres de recherche qui, plus que de s'investir dans une stratégie collective autour de projets de R\&D, (ré)activent leurs relations de lobbying par le biais des conseils d'administration des Pôles ou directement avec l'Etat central qui est, dans le cas francilien, proche géographiquement ${ }^{14}$. Dans le Pôle Cap Digital, qui est marqué par une forte part de Petites et Moyennes Entreprises (PME) du fait du tissu industriel du secteur couvert, la perspective de financements publics produit un « effet kiss cool $\gg^{15}$ auprès d'entreprises qui n'avaient pas l'habitude de se rencontrer. Mais elle ne se traduit pas par de nouveaux comportements et par la mobilisation dans la durée des acteurs économiques privés. De manière générale, les coopérations entre les entreprises et les centres de recherche publics et privés se révèlent difficiles car, outre le fait de ne pas partager les mêmes temps d'action ou enjeux, il y a aussi la crainte de partager son expertise. Après une première phase où de nombreux projets ont été présentés, on observe un palier à partir de 2007-2008, ce qui laisse à penser que les entreprises ont avant tout déstocké des projets de R\&D en attente.

\footnotetext{
${ }^{12}$ Entretien personnel avec une responsable Direction Innovation du Conseil Régional lle-de-France (2009). ${ }^{13}$ Entretiens personnels avec un responsable de la DRIRE Ile-de-France; avec un responsable de l'action économique au CG des Yvelines; avec un responsable de la politique de l'innovation à la Ville de Paris (2009). ${ }^{14}$ Entretiens personnels avec une responsable de la Direction Innovation au Conseil Régional de I'lle-deFrance et avec une conseillère régionale socialiste (2009).

${ }^{15}$ Entretiens personnels avec une responsable de la Direction Innovation au Conseil Régional de I'lle-deFrance et avec une conseillère régionale socialiste (2009).
} 
En Ile-de-France, c'est l'Etat qui apparaît aux yeux des acteurs et des responsables des Pôles comme le mieux placé pour encadrer les négociations autour des projets de R\&D pour plusieurs raisons : non seulement son rôle financier majeur avec plusieurs Pôles mondiaux bénéficiant de la majorité des financements du FUI, mais aussi du fait des rapports de concurrence entre les grands groupes ou les centres de recherche qui y voient un moyen d'obtenir un financement public pour leurs projets de R\&D ; entre ces grands groupes et les PME qui y voient, elles, un moyen d'obtenir un label reconnu internationalement et une opportunité commerciale ; et entre des collectivités territoriales puissantes économiquement et/ou politiquement. Ceci rejoint une tendance actuelle de l'Etat central à vouloir reprendre le contrôle des questions d'aménagement et de développement de la région francilienne : que cela soit dans l'industrie automobile qui est fortement présente dans la région ( $20 \%$ de l'emploi automobile français) ou avec la réforme territoriale et la volonté de transformer le mode de gouvernement du Grand Paris, on observe des tentatives de pilotage top-down de l'Etat en Ile-de-France.

\section{Les Pôles, une politique de cluster comme les autres ? Mise en perspective européenne}

\subsection{Le développement des politiques de cluster en Europe}

Une dernière manière d'évaluer la nature et les formes prises par les collaborations dans le cadre de la politique des Pôles en Ile-de-France est d'analyser ses similitudes et ses différences avec les politiques de cluster en Europe. Deux raisons justifient une telle démarche. D'une part, les politiques de cluster en Europe sont une référence explicite (et souvent citées comme des exemples de «bonnes pratiques ») dans les rapports au Gouvernement sur la politique industrielle française (Datar, 2004 ; Blanc, 2004...) ou dans les rapports d'évaluation des Pôles. D'autre part, les Pôles sont présentés comme une réponse à l'engagement pris lors du Conseil Européen de Lisbonne en 2000 par la France, avec les autres Etats européens, d'œuvrer en faveur du développement de l'économie de la connaissance ; et un outil pour bénéficier de la réorientation des fonds structurels en faveur de la compétitivité des territoires.

Depuis les travaux fondateurs de Marshall (1919) sur les districts industriels, puis sur la Troisième Italie (Bagnasco, 1976 ; Becattini, 1990) et, dans un contexte de mondialisation accélérée, sur les avantages comparatifs des régions (Porter, 1998) et sur le rôle des réseaux d'entreprises pour répondre aux défis industriels et technologiques (Saxenian, 1994), les clusters sont devenus un objet d'analyse pour montrer leurs effets économiques tant au niveau macro qu'au niveau des entreprises. Le rôle des pouvoirs publics a été en outre souligné dans le développement d'un contexte favorable à la compétitivité et l'innovation des entreprises, soit au travers d'infrastructures, d'organisations du type incubateurs, ou de financements de projets de R\&D. Nombre d'universitaires, comme l'américain Porter, ont prodigué leurs conseils auprès d'autorités politiques nationales ou régionales à travers le monde - le Pays Basque espagnol en est un exemple célèbre - sur le «meilleur » rôle que peuvent jouer les pouvoirs publics afin de soutenir le développement de clusters.

Les clusters sont devenus aussi un objet des politiques publiques en Europe. Pour les responsables politiques, le soutien au développement des clusters a été depuis les années 90 un 
outil pour répondre à différents niveaux (national ou infranational) aux problèmes industriels et d'innovation (Lagendjik et Charles, 1999). Il s'agit d'un outil « commode » : il peut porter sur une variété de dimensions des clusters pour lesquelles les économistes, sociologues ou gestionnaires voient un rôle des pouvoirs publics, et il représente selon les cas un soutien financier public plus ou moins important. En outre, il ne fait pas l'objet de controverse politique (qui serait contre le soutien à la compétitivité économique et l'innovation ?) et reste le plus souvent cantonné à un discours technique. Il ne contredit pas non plus la réglementation européenne sur le Marché Unique, qui a connu depuis 2008 un assouplissement dans l'interdiction des aides publiques directes aux entreprises (règle de Minimis). Last but not least, il constitue un outil pour les acteurs politiques afin d'afficher auprès de leurs électeurs leur engagement pour le développement de leur territoire et obtenir des fonds de la part des échelons nationaux ou européens.

Pour Raines (2002), il existe trois éléments distincts des politiques de cluster par rapport aux autres initiatives de soutien industriel : le soutien à l'innovation et à l'apprentissage au sein des clusters ; l'accent mis sur les réseaux d'entreprises plutôt que les entreprises individuelles et sur la sélection de certains de ces réseaux par rapport à d'autres; et sur les partenariats publics-privés pour la définition et la mise en œuvre de ces politiques. La comparaison permet de confirmer ce qui a été précédemment présenté : en Europe, la France se distingue bien par la centralisation de sa politique des Pôles. Que cela soit dans le cadre d'un autre Etat unitaire, l'Angleterre, dont le Gouvernement demande aux Régions d'inclure les clusters dans leur stratégie régionale économique en 2000, d'un Etat unitaire en voie de fédéralisation économique, l'Italie, où une compétence législative a été donnée aux régions dès 1993 pour soutenir les districts industriels, ou d'Etats fédéraux comme l'Allemagne, la définition et la mise en œuvre multi-niveaux des politiques de cluster est explicitement choisie (Borràs et Tsagdis, 2008 ; Kiese, 2008 ; Menu, 2009).

\subsection{L'exemple bavarois : l'apprentissage des clusters piloté par le pouvoir centralisé régional}

L'exemple de l'Etat centralisé bavarois est particulièrement intéressant à comparer avec l'expérience des Pôles en Ile-de-France. La Bavière a un statut institutionnel et politique particulier par rapport aux Länder allemands : l'existence d'un Gouvernement régional dirigé par un Ministerpräsident élu tous les quatre ans lors d'élections régionales, du fait de la structure fédérale de l'Allemagne, est renforcée par la tradition étatique (on ne parle pas du Land mais de l'Etat de Bavière) et par une culture administrative centralisée depuis le 19è siècle. En outre, la Bavière est gouvernée sans interruption depuis 1962 par le parti régionaliste de la Christlich-Soziale Union (CSU) qui y constitue l'unique parti conservateur, et qui a un accès direct aux structures fédérales par le biais de son alliance avec la CDU et du Bundesrat (Parlement des Länder). Dans le domaine industriel, une politique active est menée par le Gouvernement bavarois dès 1946 en réorientant les fonds fédéraux issus du système de péréquation et en participant directement au capital des banques et des compagnies d'assurances. En particulier, l'homme politique conservateur F-J. Strauss défend le développement industriel de la Bavière : tour à tour Ministre fédéral de la Défense, des Finances et Ministerpräsident de la Bavière entre 1956 et 1988, il promeut la cause industrielle dans une région encore fortement rurale (Milosch, 2006). Aujourd'hui, la Bavière se situe aux premiers rangs des régions européennes d'un point de vue économique et technologique. 
On retrouve donc au niveau de la Bavière la tradition étatique centralisée et interventionniste de la France dans le domaine industriel. Lorsque le Gouvernement fédéral lance une politique de cluster visant à développer les biotechnologies (Bioregionen) en 1998, puis une HighTech Strategie en 2006 pour financer une quinzaine de clusters (Spitzencluster) à hauteur de 600 millions d'euros, la Bavière a déjà ancré un solide programme de soutien aux technologies et à l'innovation. S'appuyant sur les ressources tirées des privatisations dans les secteurs de la banque et de l'assurance, le Gouvernement bavarois lance dès 1994 l'Offensive Zukunft Bayern (OZB) pour financer des instituts universitaires techniques ou de $R \& D$, ainsi que des programmes de transfert technologique dans l'industrie. En 2004, l'OZB totalise plus de quatre milliards d'euros d'investissement. En 2006, le Gouvernement bavarois recentre son action autour d'une Clusterpolitik qui soutient 19 « domaines de compétence » répartis sur l'ensemble du territoire régional (Bayerisches Staatsministerium für Wirtschaft, 2009).

Pour faciliter les collaborations entre industrie et recherche, l'agence de statut privé Bayern Innovativ est créée en 1996. Elle est présidée par le Ministre de l'économie et est dirigée depuis lors par un ancien universitaire, qui fut aussi cadre dirigeant dans une multinationale américaine. Dotée de financements conséquents, elle vise à promouvoir des réseaux de coopérations industrielles ou de transfert technologique dans différents secteurs (Menu, 2009). Depuis 2006, elle gère directement ou coordonne l'action des 19 clusters (Kiese, 2008). L'organisation du soutien bavarois aux clusters s'appuie ainsi sur les pratiques de partenariats publics-privés ancrées depuis plus d'une décennie, et sur les mêmes pivots humains comme le Directeur de Bayern Innovativ; ce qui ne l'empêche pas d'être supervisée par le ministère de l'économie bavarois et de faire l'objet d'une rhétorique politique faisant des clusters répartis sur l'ensemble du territoire bavarois les bases du développement économique régional.

Avec les politiques de cluster, la régulation politique des économies locales reste très diversifiée, comme pour d'autres initiatives publiques précédentes (Schmidt, 2008 ; Crouch et al., 2004). Dans les cas francilien et bavarois, on observe une régulation par l'Etat central, mais alors que dans le premier cas il s'agit du niveau national, en Bavière, l'action centralisée se situe au niveau régional, bénéficiant ainsi de l'atout de la proximité géographique et de la possibilité d'inclure la politique de cluster dans une stratégie plus globale de développement économique régional. Toutefois, l'exemple bavarois n'illustre pas tant l'efficacité d'un échelon d'action publique centralisé que la capacité d'individus, issu(s) des secteurs public ou/et privé, à définir un ensemble de règles et de pratiques qui guident l'action menée par des acteurs et des responsables des clusters aux intérêts très différents.

Or, ce cadre d'action publique fondé sur des règles (organisations, normes) et des pratiques partagées entre les acteurs publics et privés n'est pas explicite dans le cas francilien. Un responsable de la DRIRE de l'Ile-de-France n'affirme pas autre chose quand il fait de l'absence d'une « vision commune de l'innovation » un problème central de la mise en œuvre des Pôles dans cette région ${ }^{16}$. En Ile-de-France, il est encore difficile de voir poindre des modes de gouvernance des Pôles où acteurs publics et privés, dotés de ressources dont ils ont la maîtrise et de modes de résolution de conflits, puissent faire l'apprentissage de projets communs. En règle générale pour les Pôles, ce n'est pas tant la complexité du système de

\footnotetext{
${ }^{16}$ Entretien personnel avec un responsable de la DRIRE Ile-de-France (2009).
} 
financement et de mise en œuvre entre des acteurs pluriels (Weil et Fen Chong, 2008) qui pose problème - on la retrouve dans la plupart des Etats européens engagés dans des politiques à plusieurs niveaux et avec plusieurs types de financement (Borràs et Tsagdis, 2008) - mais bien l'incapacité à définir et à imposer une stratégie commune à laquelle chacun est ensuite tenu de se tenir, au-delà des appels à candidature au financement de projets de R\&D.

\section{Conclusion}

En 2008, l'équipe du CEPREMAP posait la question : « les Pôles de Compétitivité, que peut-on en attendre?». Cet article interroge les logiques d'action publique qui ont été à l'œuvre depuis le lancement des Pôles en 2005. Avec les Pôles, la politique industrielle de la France est de nouveau à l'agenda politique. Elle change moins dans le contenu, en confirmant le soutien microéconomique mis en œuvre depuis plusieurs années, que dans son ampleur au travers d'un financement massif de projets de R\&D, et dans ses moyens d'action, puisqu'il s'agit en priorité de projets définis et menés par des acteurs économiques privés au sein des gouvernances des Pôles. Au travers de la définition et de la mise en œuvre centralisée de cette politique, et malgré l'implication des échelons infranationaux, en particulier régionaux, on constate que l'Etat français a fait aussi et surtout des Pôles un instrument de mise en scène de son rôle comme acteur central du champ industriel, à la fois au niveau organisationnel et normatif. Or, l'Etat central n'apparaît pas comme le leader pertinent pour développer des clusters dans le temps. Déjà en 1992, Cohen voyait une incompatibilité entre la flexibilité croissante de la production, l'attractivité des territoires, l'innovation et la régulation centralisée, menée par des hauts fonctionnaires au sein de l'appareil d'Etat, de l'industrie française (Cohen, 1992b). 


\section{Bibliographie}

ALBERTINI J-B. (2007), Un exemple de réforme administrative «silencieuse » : l'accompagnement des pôles de compétitivité, Revue Française d'Administration Publique 124 (4), 673-681.

BAGNASCO A. (1976), Tre Italie. La problematica territoriale dello sviluppo italiano, Il Mulino, Bologna.

BAYERISCHES STAATSMINISTERIUM FÜR WIRTSCHAFT, Cluster Offensive Bayern, 2009.

BECATTINI G. (1990), The Marshallian industrial district as a socio-economic notion in F. PYKE, G. BECATTINI and W. SENGENBERGER (eds), Industrial districts and inter-firm co-operation in Italy, Genève, International Institute for Labour Studies.

BEFFA J-L. (2005), Pour une nouvelle politique industrielle, rapport au Président de la République, Paris, la Documentation française.

BERTHET D. et TERNISIEN M. (2004), La désindustrialisation de 1'Ile-de-France ?, Futuribles 297, 31-47.

BEZES P. (2009), Réinventer l'Etat : les réformes de l'administration française (1962-2008), Presses de Sciences Po, Paris.

BLANC C. (2004), Pour un écosystème de la croissance, Rapport au Premier ministre, La Documentation française, Paris.

BORRAS S. et TSAGDIS D. (2008), Cluster policies in Europe, Firms, Institutions, and Governance, Cheltenham, Edward Elgar.

BOSTON CONSULTING GROUP \& CM INTERNATIONAL (2008), Évaluation des Pôles de Compétitivité, rapport au Gouvernement Fillon, juin.

BREDIF H., H. (2009), Quels projet d'intérêt national pour le plateau de Saclay ?, Information géographique 3.

BRUNO I. (2008), A vos marques®, prêts... cherchez! La stratégie européenne de Lisbonne, vers un marché de la recherche, Ed. du Croquant, Bellecombe-en-Bauges.

CARAYON B. (2005), Les outils de la politique industrielle, rapport d'information de la Commission de Finances, de l'Economie Générale et du Plan, Assemblée Nationale, mai.

COHEN E. (1992a), Dirigisme, politique industrielle et rhétorique industrialiste, Revue Française de Science Politique 42 (2), 197-218.

COHEN E. (1992b), Politique industrielle ou politiques de compétitivité, Notes Saint-Simon, juin.

CONSEIL REGIONAL D'ILE DE FRANCE (2009), Bilan économique de la Région de l'Ile-de-France.

CRESPY C. (2007), Gouvernance de la recherche et compétitivité des régions : quel rôle pour l'action publique territoriale ?, Politiques et Management Public 25 (2).

CROUCH C. et al., (2001), Local Production Systems: Rise or Demise?, Oxford University Press, Oxford.

Délégation à l'Aménagement du Territoire et à l'Action Régionale (2004), La France, puissance industrielle, une nouvelle politique industrielle pour les territoires, Paris, La Documentation française/DATAR.

DURANTON G. et al.,(2008), Les Pôles de compétitivité : que peut-on en attendre ?, Collection du CEPREMAP, 10 , Edition Rue d'Ulm, Paris.

DURANTON G. et al.,(2009), Whither clusters? Lessons from the French experience, Oxford University Press, Oxford.

FEN-CHONG S. (2006), Trois réponses régionales à un processus national, Annales des Mines, Série « Réalités industrielles », 38-41.

GILLI F. et OFFNER J-M. (2009), Paris, métropole hors les murs: aménager et gouverner un Grand Paris, Presses de Sciences Po, Paris.

GOMEZ P-Y. (2009), « La gouvernance des Pôles de compétitivité. Impasses théoriques et reformulation de la spécificité des pôles », Revue Française de Gestion, n¹90, p. 197-209.

GROSSETTI M. et ZULIANI J-M. (2009), « Toulouse and the on-board systems cluster: a late result from one century of local and national policies », communication for the colloquium « Cluster policies in European regions: Governance, innovation and actor interaction », Audencia Nantes School of Management, October 
(http://www.audencia.com/la-recherche/domainesdengagementinstitutionnel/institut-entrepreneuriat/ evenements/archives-evenements/).

KIESE M. (2008), "Mind the Gap: Regional Clusterpolitik im Spannungsfeld von Wissenschaft, Politik und Praxis aus der Perspektive der Neuen Politischen Ökonomie", Zeitschrift für Wirtschaftsgeographie, 52(2-3), p. 129-145.

LAGENDJIK A. et CHARLES D. (1999), "Clustering as a new growth strategy for regional economies? A discussion of new forms of regional industrial policy in the UK", in OECD, Boosting innovation: the cluster approach, Paris, p. 127-154.

LASCOUMES P. et LE GALES P. (2006), « Instrument », in BOUSSAGUET L., JACQUOT S. et RAVINET P., Dictionnaire des politiques publiques, Paris, Presses de Sciences Po.

LEFEBVRE P. (2009), "Cluster policy in France: regions and multi-level governance in a state-led policy", communication for the colloquium «Cluster policies in European regions: Governance, innovation and actor interaction », Audencia Nantes School of Management, October (http://www.audencia.com/la-recherche/ domainesdengagementinstitutionnel/institut-entrepreneuriat/evenements/archives-evenements/).

LE GALES P. (2006), Gouvernance in BOUSSAGUET L., JACQUOT S. et RAVINET P., Dictionnaire des politiques publiques, Presses de Sciences Po, Paris.

LE LIDEC P. (2007), Le jeu du compromis. L'Etat et les collectivités territoriales dans la décentralisation en France, Revue Française d'Administration Publique 121-122, 111-130.

MARSHALL A. (1919), Industry and Trade, a Study of industrial technique and business organization, and on their influences on the conditions of various classes and nations, MacMillan, London.

MENU S. (2009), Le potentiel des identités territoriales pour l'action économique : les cas de la Bavière, de la Bretagne et du Nord-Est de l'Angleterre in GIS-GRALE, Droit et Gestion des collectivités territoriales, Editions du CNRS, Paris.

MILOSCH M. (2006), Modernizing Bavaria, the politics of Franz-Josef Strauss and the CSU, 1949-1979, Bergham Books, New York.

PASCALLON P. et HORTEFEUX P. (2008), Que faut-il penser des pôles de compétitivité ?, Revue politique et parlementaire 1047, 139-143.

PORTER M. (1998), Clusters and the new economics of competition, Harvard Business Review, 76(6), 77-90.

RAINES P. (ed.) (2002), Cluster Development and Policy, Ashgate, Burlington.

SAXENIAN A. (1994), Regional Advantage: Culture and Competition in Silicon Valley and Route 128, Mass., Harvard University Press, Cambridge.

SCHMIDT V. (1996), The Decline of Traditional State Dirigisme in France: the Transformation of Political economic policies and policy-making processes, Governance 9(4), 375-405.

SCHMIDT V. (1997), Running on empty: the end of dirigisme in French economic leadership, Modern \& Contemporary France 5 (2), 229-241.

SCHMIDT V. (2008), European Political Economy: Labour Out, State Back In, Firm to the Fore, West European Politics 31 (1-2), 302-320.

WEIL T., FEN-CHONG S. (2008), Les pôles de compétitivité français, Futuribles 342, juin, 5-26. 


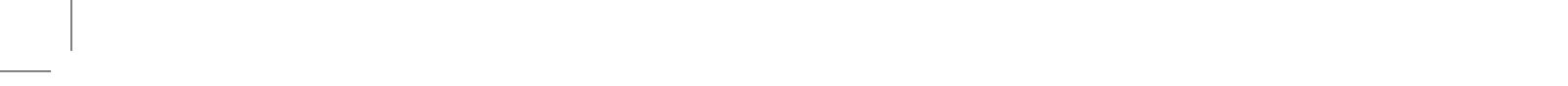

